RAIRO Operations Research

RAIRO Oper. Res. 41 (2007) 465-478

DOI: $10.1051 /$ ro:2007030

\title{
MARKET CLEARING PRICE AND EQUILIBRIA OF THE PROGRESSIVE SECOND PRICE MECHANISM
}

\author{
PATRICK MAILlÉ ${ }^{1}$
}

\begin{abstract}
The Progressive Second Price mechanism (PSP), recently introduced by Lazar and Semret to share an infinitely-divisible resource among users through pricing, has been shown to verify very interesting properties. Indeed, the incentive compatibility property of that scheme, and the convergence to an efficient resource allocation where established, using the framework of Game Theory. Therefore, that auction-based allocation and pricing scheme seems particularly wellsuited to solve congestion problems in telecommunication networks, where the resource to share is the available bandwidth on a link. This paper aims at supplementing the existing results by highlighting some properties of the different equilibria that can be reached. We precisely characterize the possible outcomes of the PSP auction game in terms of players bid price: when the bid fee (cost of a bid update) tends to zero then the bid price of all users at equilibrium gets close to the socalled market clearing price of the resource. Therefore, observing an equilibrium of the PSP auction game gives some accurate information about the market clearing price of the resource.
\end{abstract}

Keywords. Network pricing, Game theory, Auctions.

Mathematics Subject Classification. 91A, 91B.

\section{INTRODUCTION}

Network pricing is encountering a growing interest from resource providers, since it appears as a solution against congestion. Indeed, with the appearance of new

Received May 19, 2005. Accepted May 7, 2007.

${ }^{1}$ GET/ENST Bretagne, 2 Rue de la Châtaigneraie, CS 17607, 35576 Cesson Sévigné Cedex, France; patrick.maille@enst-bretagne.fr

(c) EDP Sciences, ROADEF, SMAI 2007

Article published by EDP Sciences and available at http://www.rairo-ro.org or http://dx.doi.org/10.1051/ro:2007030 
services that need high communication rates (video on demand) and the soaring of the number of users, situations of network saturation occur more and more frequently. Therefore, pricing mechanisms that constitute an incentive to regulate consumption may replace in the future the current flat-rate schemes, with which congestion is more likely to happen. The recent literature on the subject shows the importance of developping new allocation and pricing mechanisms: many papers propose pricing solutions, for wireless $[1,16]$ and wired networks $[6,8,10,11,18]$.

The objective in both cases is to fairly share the resources (bandwidth, computing power) among users. The notions we use here - fairness, optimal allocation, user reaction to a pricing mechanism - come from economics, which provides a theoretical framework to study the performance of a system. In particular, the formalism of non-cooperative game theory is well-suited to describe the behavior of users and resource sellers (the players in the allocation game), since the potential users of a network, competing for the resource, can be considered as behaving selfishly.

Using this formalism, Lazar and Semret recently introduced the Progressive Second Price mechanism (PSP) [9, 17], an auction-based scheme to allocate bandwidth among users: players can modify their bid as a reply to the bids submitted by the others (with a bid change fee $\varepsilon$ ), and allocations and prices are computed following second-price auction principles.

Lazar and Semret prove that players are incentivized to bid truthfully, and that the auction game converges after a finite time to an $2 \varepsilon$-Nash equilibrium, i.e. a situation where no player (user) can improve his utility by more than a certain value $2 \varepsilon>0$ by changing his bid. Moreover, that equilibrium corresponds to an efficient resource allocation in the sense that it maximizes social welfare.

In this paper, we focus on the structure of the equilibrium reached by the PSP auction game. We link that equilibrium to the users' willingness-to-pay functions, and illustrate how that result could be used by the seller in order to maximize his revenue (notice that it is natural to consider that the seller will try to obtain the highest revenue: see $[13,14]$ for results in the case of a single item to be sold).

The paper is organized as follows. Section 1 presents the PSP mechanism suggested by Lazar and Semret and quote their main results. In Section 2, we define the market clearing price as the unit price for which the aggregated demand equals the total available resource, and we characterize all Nash equilibria of the game where players (users) reveal their true valuations. We establish the relation between such equilibria, called truthful, and the market clearing price. Since the PSP scheme leads to $2 \varepsilon$-Nash equilibria, we investigate in Section 3 all truthful such equilibria for small values of $\varepsilon$, and also relate them to the market clearing price. Conclusions and future work are presented in Section 4. 


\section{THE PROGRESSIVE SECOND PRICE MECHANISM}

We describe here the PSP allocation and pricing mechanism for an infinitely divisible resource (bandwidth on a communication $\operatorname{link}^{1}[9]$ ).

In order to share the total amount of available resource $Q$, among a set $\mathcal{I}=$ $\{1, \ldots, I\}$ of players, Lazar and Semret suggest to use the following auction-based scheme:

- each player $i$ submits a 2-dimensional bid $s_{i}=\left(q_{i}, p_{i}\right) \in \mathcal{S}_{i}:=[0, Q] \times$ $[0,+\infty)$, where $q_{i}$ is a desired quantity of resource and $p_{i}$ the unit price player $i$ is willing to pay for that resource. A bid $s_{0}:=\left(Q, p_{0}\right)$ is introduced by the owner of the resource to ensure that the bandwidth is not sold at a unit price below $p_{0}$, that is called the reserve price, we therefore define $\mathcal{I}_{0}:=\mathcal{I} \cup\{0\}$ where player 0 is the resource owner;

- allocated quantities of resource $\left(a_{i}\right)_{i \in \mathcal{I}_{0}}$, and prices $\left(c_{i}\right)_{i \in \mathcal{I}}$ charged to players are computed based on the bid profile $s:=\left(s_{1}, \ldots, s_{I}\right)$ : the resource goes first to players with the highest prices in their bids until it is completely allocated. The pricing rule is chosen such that the total charge $c_{i}$ of player $i$ covers the "social opportunity cost" of his presence, i.e. the declared willingness-to-pay of players who are excluded by player $i$ 's bid. In that sense, the PSP scheme is an extension of Vickrey auctions [19], and is inspired by Clarke-Groves mechanisms $[4,7]$.

More formally, the resource allocation $a_{i}(s)$ that player $i$ obtains and the price $c_{i}(s)$ he is charged when the bid profile is $s$ are given by

$$
\begin{aligned}
a_{i}(s) & :=q_{i} \wedge \underline{Q}_{i}\left(p_{i}, s_{-i}\right), \\
c_{i}(s) & :=\sum_{j \neq i} p_{j}\left[a_{j}\left(s_{-i}\right)-a_{j}(s)\right],
\end{aligned}
$$

where $s_{-i}:=\left(s_{1}, \ldots, s_{i-1}, s_{i+1}, \ldots, s_{I}\right)$ is the bid profile that player $i$ faces, i.e. $s=\left(s_{i} ; s_{-i}\right)$, and

$$
\underline{Q}_{i}\left(p_{i}, s_{-i}\right):=\left[Q-\sum_{p_{k} \geq p_{i}, k \neq i} q_{k}\right]^{+}
$$

is the amount of resource remaining after all players with price larger than $p_{i}$ are allocated their asked quantity (i.e. the maximum allocation that player $i$ can expect by bidding price $p_{i}$ when faced with the bid profile $\left.s_{-i}\right)$. The allocation $a_{j}\left(s_{-i}\right)$ corresponds to the allocation that player $j$ would have obtained according to the allocation rule (1) if player $i$ had not been present in the game, i.e. if the bid profile had been $s_{-i}$ instead of $s$.

\footnotetext{
${ }^{1}$ Lazar and Semret also studied an extension of PSP to a whole network, but we focus in this paper on the case of a single link.
} 


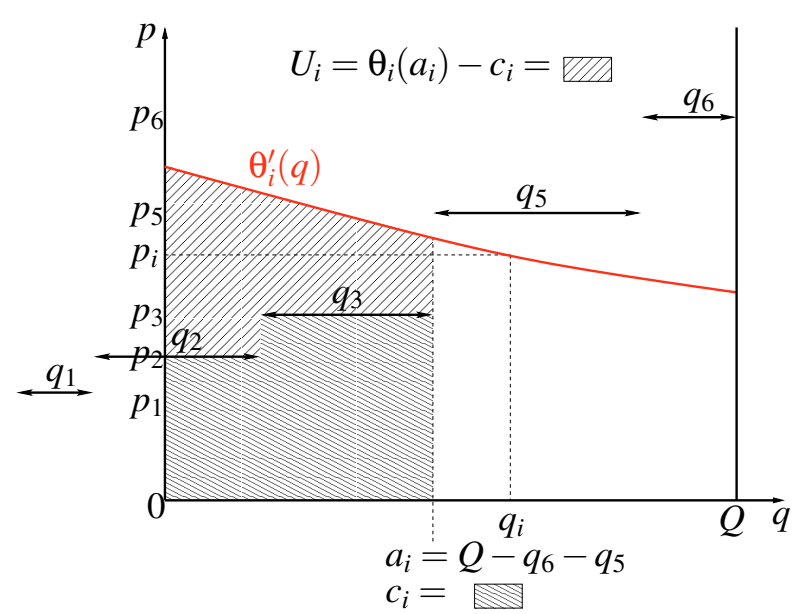

Figure 1. Allocation and price to pay for player $i$ with bid $s_{i}=$ $\left(q_{i}, p_{i}\right)$, and corresponding utility $U_{i}$.

User preferences are modelled by quasilinear utility functions, of the form $U_{i}(s):=\theta_{i}\left(a_{i}(s)\right)-c_{i}(s)$, where $\theta_{i}\left(a_{i}\right)$ is the maximum price player $i$ is willing to pay to obtain allocation $a_{i}\left(\theta_{i}\right.$ is called the valuation function of user $i$, assumed nondecreasing, differentiable, and such that $\left.\theta_{i}(0)=0\right)$.

The PSP allocation and pricing rules, and the associated value of player $i$ 's utility, are illustrated in Figure 1.

The auction game works as follows: each player $i \in \mathcal{I}$ can make a bid $s_{i}$ (or change his bid) each $T$ units of time, knowing perfectly the bids made by the other players $s_{-i}$. There is a bid fee $\varepsilon$ charged for each bid change. Moreover, it is assumed that two players never bid at exactly the same time.

Defining a truthful bid as a bid $s_{i}=\left(q_{i}, p_{i}\right)$ such that $p_{i}=\theta_{i}^{\prime}\left(q_{i}\right)$ (that is, bidding a price equal to the marginal valuation), Lazar and Semret prove (see [9], Prop. 1) that submitting truthful bids is profitable for players (incentive compatibility property). Consequently, they suggest a truthful behavior for players, which ensures the convergence of bids in a finite time to a truthful $2 \varepsilon$-Nash equilibrium, i.e. a bid profile where no player can increase his utility by more than $2 \varepsilon$ by unilaterally changing his bid [9]. Moreover, the corresponding allocation is efficient in the sense of the social welfare $\sum_{i \in \mathcal{I} \cup\{0\}} \theta_{i}\left(a_{i}\right)$ with $\theta_{0}(q):=p_{0} q$ (see [17], Prop. 3).

To derive those theoretical results, Lazar and Semret assume that the valuation functions verify some regularity properties, that we also consider in this paper:

Assumption 1.1. For any $i \in \mathcal{I}$,

- $\theta_{i}$ is differentiable, and $\theta_{i}(0)=0$;

- $\theta_{i}^{\prime} \geq 0$, non-increasing and continuous;

- $\exists \gamma_{i}>0, \forall z \geq 0, \theta_{i}^{\prime}(z)>0 \Rightarrow \forall \eta<z, \theta_{i}^{\prime}(z) \leq \theta_{i}^{\prime}(\eta)-\gamma_{i}(z-\eta)$. 
Assumption 1.2. $\exists \kappa>0, \forall i \in \mathcal{I}$,

- $\forall y, z, y>z \geq 0, \theta_{i}^{\prime}(y)-\theta_{i}^{\prime}(z)>-\kappa(y-z)$;

- $\theta_{i}^{\prime}(0)<+\infty$.

Remark 1.3. Assumption 1.1 can be interpreted as follows: more resource means more value for a user, but the valuation of obtaining one more resource unit decreases with the allocation, since its relative impact on performance decreases. The parameter $\gamma_{i}$ gives a lower bound on this decreasingness (or equivalently on the concavity of $\theta_{i}$ ): while it is strictly positive, the marginal valuation of resource decreases faster than $-\gamma_{i}$. Likewise, Assumption 1.2 imposes an upper bound on the decreasingness of the marginal valuation function: $\theta_{i}^{\prime}$ decreases more slowly than $-\kappa$. Notice that we necessarily have $\gamma_{i} \leq \kappa$ for all $i \in \mathcal{I}$. When the valuation functions of all users are piecewise twice differentiable, then we can satisfy both assumptions by setting $\gamma_{i}:=\sup _{q: \theta_{i}^{\prime}(q)>0} \theta_{i}^{\prime \prime}(q)$ for all $i \in \mathcal{I}$ and $\kappa:=\inf _{i, q} \theta_{i}^{\prime \prime}(q)$. In the particular case when all users have the same valuation function that is parabolic in its increasing part, i.e. the marginal valuation function is of the form $\theta_{i}^{\prime}(q)=\left[\theta_{i}^{\prime}(0)-\gamma_{i} q\right]^{+}$, then $\gamma_{i}=\kappa$ for all users.

\section{Market Clearing price and truthful NaSh EQUilibria}

In this section, we define the market clearing price and the truthful Nash equilibria of the PSP auction game, and prove that those notions are intimately linked. To do so, we first introduce the demand function $d_{i}$ of a user $i \in \mathcal{I}$.

Definition 2.1. For $i \in \mathcal{I}$, define the demand function of player $i$ as the function $d_{i}$ such that for every unit price $p>0$,

$$
d_{i}(p)=\arg \max _{q}\left\{\theta_{i}(q)-p q\right\} .
$$

$d_{i}(p)$ thus corresponds to the resource quantity that player $i$ would want to buy in order to maximize his utility, if the unit price of the resource were fixed to $p$.

Assumption 1.1 ensures the existence and uniqueness of $d_{i}(p), \forall p>0$. Indeed, we have $d_{i}(p)=\left\{\begin{array}{ll}\left(\theta_{i}^{\prime}\right)^{-1}(p) & \text { if } 0<p \leq \theta_{i}^{\prime}(0) \\ 0 & \text { if } p>\theta_{i}^{\prime}(0)\end{array}\right.$. (Remark that $\forall q, \theta_{i}^{\prime}(q)>0 \Rightarrow$ $\left.d_{i}\left(\theta_{i}^{\prime}(q)\right)=q.\right)$

Definition 2.2. Under Assumptions 1.1 and 1.2, all demand functions $\left(d_{i}\right)_{i \in \mathcal{I}}$ are continuous and non-increasing on $(0,+\infty[$, and they are strictly decreasing as long as they take strictly positive values.

The market clearing price is then the unique price $u$ (if it exists) such that

$$
\sum_{i \in \mathcal{I}} d_{i}(u)=Q
$$

We also define $\mathcal{I}^{+}:=\left\{i \in \mathcal{I} \mid d_{i}(u)>0\right\}$, i.e. the set of players that would buy a strictly positive quantity of resource if the unit price were $u$. 
Remark 2.3. Those concepts of demand function and market clearing price are common in economics. They are also more and more used to study telecommunication systems, as for example in $[5,12]$. We straightforwardly obtain a condition for the existence of the market clearing price:

Lemma 2.4. A necessary and sufficient condition for the market clearing price to exist and be strictly greater than $p_{0}$ is that

$$
\sum_{i \in \mathcal{I}} d_{i}\left(p_{0}\right)>Q
$$

We now define the notion of Nash equilibrium. In game theory, a Nash equilibrium is a configuration of player strategies such that nobody can improve his utility by unilaterally changing his strategy. When they exist, Nash equilibria are generally expected to represent the possible outcomes of noncooperative games. In our context Nash equibria can be formally defined as follows.

Definition 2.5. A Nash equilibrium of the PSP game is a bid profile $s=\left(s_{1}, \ldots, s_{I}\right)$ such that

$$
\forall i \in \mathcal{I}, \forall \tilde{s}_{i}=\left(\tilde{q}_{i}, \tilde{p}_{i}\right) \in \mathcal{S}_{i}, \quad U_{i}\left(\tilde{s}_{i}, s_{-i}\right) \leq U_{i}(s)
$$

i.e. no player can be better off by unilaterally changing his bid.

Moreover, we say that a Nash equilibrium is truthful if all players submit a truthful bid, i.e. $p_{i}=\theta_{i}^{\prime}\left(q_{i}\right), \forall i \in \mathcal{I}$.

Remark 2.6. The truthful bidding strategy pointed out by Lazar and Semret leads to a $2 \varepsilon$ truthful Nash equilibrium. However, Nash equilibria of the PSP game are not necessarily truthful: assume for example that the marginal valuation of player 1 is such that $\theta_{1}^{\prime}(Q)>p_{0}$. Then it is easy to see from the PSP allocation and pricing rules that the bid profile such that $s_{1}=\left(Q, p_{1}\right)$ with $p_{1}>\max _{i \neq 1} \theta_{i}^{\prime}(0)$ and $s_{i}=(0,0)$ for all $i \neq 1$ is a Nash equilibrium: player 1 gets all the resource and pays a unit price $p_{0}$, while no other player could obtain some resource at a unit price below $p_{1}$.

In this paper, we consider that users follow a myopic truthful bid update strategy like the one proposed by Lazar and Semret. We therefore focus only on truthful Nash (and $\varepsilon$-Nash) equilibria of the PSP game.

We now characterize all truthful Nash equilibria of the PSP auction game, using the market clearing price $u$ :

Proposition 2.7. If (6) holds, then under Assumptions 1.1 and 1.2, the truthful Nash equilibria of the PSP game are the bid profiles $s^{*}$ such that all users that effectively obtain some resource bid the same unit price $p_{i}^{*}=u$ with $u$ the market clearing price.

More precisely, Condition (6) implies that every bid profile $s^{*}$ such that

$$
\begin{cases}\forall i \in \mathcal{I}^{+}, & s_{i}^{*}=\left(d_{i}(u), u\right) \\ \forall i \in \mathcal{I} \backslash \mathcal{I}^{+}, & s_{i}^{*}=\left(q_{i}^{*}, \theta_{i}^{\prime}\left(q_{i}^{*}\right)\right)\end{cases}
$$


is a truthful Nash equilibrium for the PSP auction game; and under Assumptions 1.1 and 1.2, $(N)$ is also a necessary condition for a bid profile to be a truthful Nash equilibrium.

Proof. Define (as in [17]) for all bid profile $s$ :

$Q_{i}\left(y, s_{-i}\right):=\left[Q-\sum_{j \neq i: p_{j}>y} q_{j}\right]^{+}$and $P_{i}\left(z, s_{-i}\right):=\inf \left\{y \geq 0: z \leq Q_{i}\left(y, s_{-i}\right)\right\}$

which implies that $c_{i}(s)=\int_{0}^{a_{i}(s)} P_{i}\left(z, s_{-i}\right) \mathrm{d} z$, and that

$$
\forall y, z \quad z \leq Q_{i}\left(y, s_{-i}\right) \Leftrightarrow P_{i}\left(z, s_{-i}\right) \leq y .
$$

We need to show that when the bid profile is $s^{*}$, no player $i \in \mathcal{I}$ could improve his utility by unilaterally changing his bid. First note that the allocations $\left(a_{i}^{*}\right)_{i \in \mathcal{I}}$ can be computed: since $\forall i \in \mathcal{I}, p_{i}^{*} \leq u$ and $\underline{Q}_{i}\left(., s_{-i}^{*}\right)$ is non-decreasing, we have

$$
\underline{Q}\left(p_{i}^{*}, s_{-i}^{*}\right) \leq \underline{Q}\left(u, s_{-i}^{*}\right)=Q-\sum_{j \neq i} d_{j}(u)=d_{i}(u),
$$

which proves that $a_{i}^{*}=d_{i}(u)$ (see Eq. (1)).

Assume that there exist $r>0$ and $\eta>0$ such that $P_{i}\left(a_{i}^{*}+r, s_{-i}^{*}\right) \leq u-\eta$. (7) would then imply that $a_{i}^{*}+r \leq Q_{i}\left(u-\eta, s_{-i}^{*}\right) \leq Q-\sum_{j \neq i, j \in \mathcal{I}^{+}} d_{j}(u)=a_{i}^{*}$, which leads to a contradiction, therefore

$$
\forall r>0, P_{i}\left(a_{i}^{*}+r, s_{-i}^{*}\right) \geq u \geq P_{i}\left(a_{i}^{*}, s_{-i}^{*}\right),
$$

where the last inequality stems from the fact that $a_{i}^{*} \leq \underline{Q}_{i}\left(u, s_{-i}^{*}\right) \leq Q_{i}\left(u, s_{-i}^{*}\right)$ and from (7). We can now compare the utility $U_{i}^{*}$ that a player $i \in \mathcal{I}$ gets in the current situation, with the utility $\tilde{U}_{i}$ he would receive by changing his bid (we denote by $\tilde{a}_{i}$ the corresponding allocation):

$$
U_{i}^{*}-\tilde{U}_{i}=\theta_{i}\left(a_{i}^{*}\right)-\theta_{i}\left(\tilde{a}_{i}\right)-\int_{\tilde{a}_{i}}^{a_{i}} P_{i}\left(z, s_{-i}^{*}\right) \mathrm{d} z \geq\left(\theta_{i}^{\prime}\left(a_{i}^{*}\right)-u\right)\left(a_{i}^{*}-\tilde{a}_{i}\right)
$$

where we used the concavity of $\theta_{i}$, Equation (8) and the non-decreasingness of $P_{i}\left(., s_{-i}^{*}\right)$. Finally we have

- if $i \in \mathcal{I}^{+}$then $\theta_{i}^{\prime}\left(a_{i}^{*}\right)=u$, and (9) gives $U_{i}^{*}-\tilde{U}_{i} \geq 0$;

- if $i \notin \mathcal{I}^{+}$, then $\theta_{i}^{\prime}\left(a_{i}^{*}\right) \leq u$ and $a_{i}^{*}=d_{i}(u)=0 \leq a_{i}^{\prime}$, so $U_{i}^{*}-\tilde{U}_{i} \geq 0$.

Therefore none of the players, facing the others' bids, has an interest to modify his own bid: the bid profile $s^{*}$ is a truthful Nash equilibrium of the PSP game.

The fact that $(N)$ is necessary under Assumptions 1.1 and 1.2 will be proved later in this paper, as a consequence of Proposition 3.1. 
Remark 2.8. Under condition (6), $(N)$ implies that $\forall i \in \mathcal{I}, p_{0} \leq P_{i}\left(q_{i}^{*}, s_{-i}^{*}\right) \leq u$, so the seller revenue $R:=\sum_{i \in \mathcal{I}} c_{i}$ verifies $p_{0} Q \leq R \leq u Q$. Moreover, when $\mathcal{I}^{+}=\mathcal{I}$, we have $R=p_{0} Q$. Therefore the seller has no guarantee of obtaining the best revenue possible from the resource, as pointed out in [3]. It could therefore be interesting for the seller to put a reserve price close below the market clearing price in order to maximize his revenue.

\section{3. $\varepsilon$-NASH EQUILIBRIUM AND MARKET CLEARING PRICE}

In this section, we establish that for each truthful $\varepsilon$-Nash equilibrium of the game, the prices $p_{i}$ submitted by players in $\mathcal{I}^{+}$(i.e. the players that effectively get some resource) are close to the market clearing price for small values of $\varepsilon$.

Proposition 3.1. Assume Assumptions 1.1 and 1.2 and inequality (6) hold (thus the market clearing price $u$ exists and is larger than $p_{0}$ ). Then for all truthful $\varepsilon$-Nash equilibrium $s$ with a sufficiently small $\varepsilon$, all players who obtain some resource have a bid price close to the market clearing price $u$, the distance between those prices being upperbounded by a value only depending on $\varepsilon$ and users utility parameters. More precisely, we have

$$
\begin{aligned}
\varepsilon<\min _{i, j \in \mathcal{I}^{+}}\left\{\frac{\gamma_{i}}{8}\left[d_{j}(u)\right]^{2}\right\} & \Rightarrow \quad \max _{i \in \mathcal{I}^{+}}\left|u-p_{i}\right| \leq 2 C \sqrt{\varepsilon} \\
& \text { with } \quad C=\sqrt{2} \kappa \max _{i \in \mathcal{I}^{+}}\left\{\frac{1}{\sqrt{\gamma_{i}}}\right\} .
\end{aligned}
$$

Proof. Since the proof is technical and uses several intermediate lemmas, it is reported to the Appendix.

Remark 3.2. This result can now be used to prove that $(N)$ is a necessary condition (see Prop. 2.7), as a truthful Nash equilibrium is a truthful $\varepsilon$-Nash equilibrium for all $\varepsilon>0$.

Proposition 3.1 proves that prices in the bids of a truthful $\varepsilon$-Nash equilibrium are arbitrarily close to the ones in a real Nash equilibrium, that we have characterized in Section 2. In particular, the bidding algorithm proposed by Semret in [9] will lead to a price profile such that $\max _{i \in \mathcal{I}^{+}}\left|u-p_{i}\right|=O_{\varepsilon \rightarrow 0}(\sqrt{\varepsilon})$.

Those results can also be used to obtain an estimation of the market clearing price based on the observation of the bid profile when an $\varepsilon$-Nash equilibrium is reached:

Proposition 3.3. Consider a truthful $\varepsilon$-Nash equilibrium s. Under Assumptions 1.1, 1.2 and inequality (6), and for a sufficiently small bid fee $\varepsilon$, the distance from the maximum unit price in the bid profile s to the market clearing price $u$ is upperbounded by a value depending on $\varepsilon$ and the user valuation parameters $\left(\gamma_{i}\right)_{i \in \mathcal{I}}$ and $\kappa$, that tends to 0 as $\varepsilon$ tends to 0 . 
Formally, if we define $\hat{u}:=\max _{i \in \mathcal{I}} p_{i}$ then

$$
|u-\hat{u}| \leq 2 C \sqrt{\varepsilon},
$$

where $C$ is given in (11).

Proof. Remark that $\max _{i \in \mathcal{I} \backslash \mathcal{I}+} p_{i} \leq u$, and apply Proposition 3.1.

Remark 3.4. This last result suggests that PSP provides an approximation of the market clearing price $u$. From Remark 1.3, the precision of the estimation depends both on user heterogeneity (via $\kappa$ that is the upper bound of the concavity of all users' valuation functions) and on the form of user valuation functions (the closer $\theta_{i}$ is to a parabole in its increasing part, the smaller the ratio of the maximum concavity to the minimum concavity of the valuation function). The best precision is attained when all users have an affine marginal valuation function of the form $\theta_{i}^{\prime}(q)=\left[\theta_{i}^{\prime}(0)-\kappa q\right]^{+}$: in that case $C=\sqrt{2 \kappa}$ and $|u-\hat{u}| \leq \sqrt{\varepsilon \times 8 \kappa}$.

\section{Conclusions and Perspectives}

In this paper, we have complemented the study of the truthful equilibria of the PSP scheme. We have introduced the market clearing price, which is a crucial notion in economics, and linked it to the Nash equilibria of that auction game. As the PSP described by Lazar and Semret actually leads to $\varepsilon$-Nash equilibria, we have proved that prices in such equilibria are still close to the market clearing price.

During our study, we have highlighted the fact that different Nash equilibria (and $\varepsilon$-Nash equilibria) may exist for a fixed set of players. This hazard does not seem desirable, and future work could be done, in order to obtain a mechanism that would lead to a unique equilibrium. A challenging work would also be to extend the results obtained in this paper for a single link to the case of a whole network with several links, where players compete for bandwidth on different routes. Semret proves in [17] that in that case the PSP auction game also converges to an $\varepsilon$-Nash equilibrium with an efficient allocation, but the structure of the equilibrium bid profile is not characterized.

The temporal aspect of the allocation game is also important: while the PSP scheme allocates resource in real-time without reservation, the perceived quality of service (and thus willingness-to-pay) often depends on the fluctuations of allocations and prices. Allowing resource reservation, as what is proposed in [15] for Walrasian auctions, would constitute a challenging extension of the model.

Another interesting extension of the model would be to consider the reserve price $p_{0}$ of the seller as a strategic variable. Indeed, as pointed out in Remark 2.8, the only guarantee for the resource owner is that the unit selling price exceeds the reserve price $p_{0}$. However, setting this reserve price to a high level reduces the probability that all the resource be sold. A trade-off then has to be found, and the results of this paper suggest that using the information contained in bids may help optimize that trade-off: for a sufficiently small bid fee $\varepsilon$, all the resource 
is sold at equilibrium when the reserve price is below the market clearing price $u$. A possible strategy for the seller could therefore be to first set a low reserve price and let the auction game converge, then to change that reserve price to a value close to $\hat{u}$ in order to sell the resource at the highest unit price possible and ensure a revenue close to $u \times Q$. Nevertheless, adjusting the reserve price close to the estimated market clearing price can be profitable for the seller only if players are not able to predict that change. Otherwise, this information should be taken into account by the players to determine their strategies and the form of the game changes, because players would then be reluctant to reveal their valuation for the resource. For example, if the seller updates its reserve price during the bidding process, as was suggested in [2], the users incentives change and determining the equilibria of the game becomes more difficult.

\section{Appendix: Proof of Proposition 3.1}

To establish Proposition 3.1, we prove several intermediate results. First, we give some inequalities that will be useful in the rest of the demonstrations:

Lemma 4.1. Under Assumptions 1.1 and 1.2, the following relations hold $\forall i \in \mathcal{I}$ :

$$
\begin{array}{ll}
\forall a, b: 0 \leq a \leq b \leq Q, & \theta_{i}(b)-\theta_{i}(a) \geq \theta_{i}^{\prime}(b)(b-a)+\frac{\gamma_{i}(b-a)^{2}}{2} \\
\forall e, f: 0<e \leq f \leq \theta_{i}^{\prime}(0), \quad & d_{i}(e)-d_{i}(f) \geq \frac{f-e}{\kappa} .
\end{array}
$$

Proof. (12) comes from Assumption 1.1 and the application of Taylor-Lagrange formula, and (13) is a consequence of Assumption 1.2 after a variable change.

Now we give a first result concerning the equilibrium allocations and prices:

Lemma 4.2. Assume that the bid profile $s$ is truthful and constitutes an $\varepsilon$-Nash equilibrium, and that the market clearing price $u$ exists and is strictly greater than $p_{0}$ (condition (6) holds). Under Assumptions 1.1 and 1.2, we have $\forall i \in \mathcal{I}$

$$
\begin{aligned}
a_{i} & \geq d_{i}(u)-\sqrt{\frac{2 \varepsilon}{\gamma_{i}}} \\
\text { and } \quad p_{i} & \leq u+\kappa \sqrt{\frac{2 \varepsilon}{\gamma_{i}}} .
\end{aligned}
$$

Proof. First notice that, since the demand functions $\left(d_{k}\right)_{k \in \mathcal{I}}$ are non-increasing and $\underline{Q}_{i}\left(., s_{-i}\right)$ is non-decreasing:

$$
\forall p \geq u, \quad \underline{Q}_{i}\left(p, s_{-i}\right) \geq \underline{Q}_{i}\left(u, s_{-i}\right) \geq[\underbrace{}_{j \in \mathcal{I}^{+}, j \neq i} d_{j}(u)]^{+} \geq d_{i}(u) \geq d_{i}(p) .
$$


This means that a player $i \in \mathcal{I}$ who submits bid $\left(d_{i}(p), p\right)$ with $p \geq u$ is ensured to obtain his asked quantity.

The lemma is trivial for $i \notin \mathcal{I}^{+}$, since in that case $d_{i}(u)=0$ and $p_{i} \leq u$, so we assume here that $i \in \mathcal{I}^{+}$.

Denote by $U_{i}$ the utility perceived by player $i$ with the current bid profile $s$, and $\tilde{U}_{i}(\tilde{t})$ (resp. $\tilde{a}_{i}$ ) the utility (resp. the allocation) that player $i$ would obtain if he changed his bid to submit the bid $\tilde{t}=\left(\tilde{q}_{i}, \tilde{p}_{i}\right)$, the bids $s_{-i}$ of the other players remaining unchanged. Assume $a_{i} \leq d_{i}(u)$ (otherwise (14) is trivial). We choose $\tilde{t}:=\left(d_{i}(u), u\right)$. (16) implies $\underline{Q}_{i}\left(u, s_{-i}\right) \geq d_{i}(u)$, so $\tilde{a}_{i}=d_{i}(u)$, and (7) gives $P_{i}\left(d_{i}(u), s_{-i}\right) \leq u$. We therefore have:

$$
\begin{aligned}
\tilde{U}_{i}\left(\left(d_{i}(u), u\right)\right)-U_{i} & =\theta\left(d_{i}(u)\right)-\theta\left(a_{i}\right)-\int_{a_{i}}^{d_{i}(u)} P_{i}\left(z, s_{-i}\right) \mathrm{d} z \\
& \geq \theta\left(d_{i}(u)\right)-\theta\left(a_{i}\right)-u\left(d_{i}(u)-a_{i}\right) \geq \gamma_{i}\left(d_{i}(u)-a_{i}\right)^{2} / 2
\end{aligned}
$$

where we used (12) and the fact that $\theta_{i}^{\prime}\left(d_{i}(u)\right)=u$ for $i \in \mathcal{I}^{+}$. The $\varepsilon$-Nash equilibrium condition implies that $\forall \tilde{t}, \tilde{U}_{i}(\tilde{t})-U_{i} \leq \varepsilon$, so $\gamma_{i}\left(d_{i}(u)-a_{i}\right)^{2} / 2 \leq \varepsilon$, which gives (14).

Relation (15) is obviously verified when $p_{i}<u$. If $p_{i} \geq u$ then (16) ensures that $a_{i}=d_{i}\left(p_{i}\right)$, and Relations (13) and (14) imply (15) (the inequality $p_{i} \leq \theta_{i}^{\prime}(0)$ stems from the fact that bids are truthful).

Lemma 4.3. Consider a truthful $\varepsilon$-Nash equilibrium. Let $\left|\mathcal{I}^{+}\right|$denote the cardinal of $\mathcal{I}^{+}$, and $\bar{p}:=\max _{i \in \mathcal{I}^{+}} p_{i}$. If $\left|\mathcal{I}^{+}\right|>1$, then for $\varepsilon<\min _{i \in \mathcal{I}^{+}}\left\{\frac{\gamma_{i}}{2}\left[d_{i}(u)\right]^{2}\right\}$,

$$
\bar{p} \geq u-\sqrt{\varepsilon} \frac{\kappa}{\left|\mathcal{I}^{+}\right|-1} \sqrt{\frac{2}{\min _{i \in \mathcal{I}^{+}} \gamma_{i}}} .
$$

Proof. The result is trivial if $\bar{p} \geq u$. We thus focus on the case $\bar{p}<u$. The quantity $q_{k}$ asked by each player $k \in \mathcal{I}^{+}$verifies:

$$
q_{k}=d_{k}\left(p_{k}\right) \geq d_{k}(\bar{p}) \geq\left(d_{k}(u)+\frac{u-\bar{p}}{\kappa}\right)
$$

where the last inequality comes from (13), with $e:=\bar{p}$ and $f:=u$ (the inequality $u<\theta_{k}^{\prime}(0)$ holds since $\left.k \in \mathcal{I}^{+}\right)$.

Now consider one player $i \in \mathcal{I}^{+}$such that $p_{i}=\min _{k \in \mathcal{I}^{+}} p_{k}$. From (1) we have

$$
a_{i} \leq\left[Q-\sum_{p_{k} \geq p_{i}, k \neq i, k \in \mathcal{I}^{+}} d_{k}\left(p_{k}\right)\right]^{+}=\left[Q-\sum_{k \neq i, k \in \mathcal{I}^{+}} d_{k}\left(p_{k}\right)\right]^{+} .
$$


Applying then (5) and (18), we obtain

$$
a_{i} \leq\left[d_{i}(u)-\left(\left|\mathcal{I}^{+}\right|-1\right) \frac{u-\bar{p}}{\kappa}\right]^{+} .
$$

But (14) indicates that $a_{i}>0$ for a sufficiently small $\varepsilon\left(\varepsilon<\frac{\gamma_{i}}{2}\left[d_{i}(u)\right]^{2}\right)$, and consequently the right-hand term in (19) is strictly positive. As a result, from lemma 4.2 we have $d_{i}(u)-\sqrt{\frac{2 \varepsilon}{\gamma_{i}}} \leq a_{i} \leq d_{i}(u)-\left(\left|\mathcal{I}^{+}\right|-1\right) \frac{u-\bar{p}}{\kappa}$, which implies $\left(\left|\mathcal{I}^{+}\right|-1\right) \frac{u-\bar{p}}{\kappa} \leq \sqrt{\frac{2 \varepsilon}{\gamma_{i}}}$ and establishes the lemma.

Lemma 4.4. Consider a truthful $\varepsilon$-Nash equilibrium. If there exist $i, j \in \mathcal{I}^{+}$such that $p_{i}<p_{j}$ and $p_{i}<u$, then for a sufficiently small $\varepsilon\left(\varepsilon<\frac{\min \left(\gamma_{i}, \gamma_{j}\right)}{8}\left[d_{i}(u)\right]^{2}\right)$,

$$
p_{j}-p_{i} \leq \kappa \sqrt{\frac{2 \varepsilon}{\gamma_{j}}}
$$

Proof. Lemma 4.2 implies that $a_{i}>\frac{1}{2} d_{i}(u)>0$, therefore $a_{j}=d_{j}\left(p_{j}\right)$ (player $j$ bids at a higher price than $i$, and thus obtains his asked quantity). Since $Q_{i}\left(p_{i}, s_{-i}\right) \geq a_{i}$ we have

$$
Q_{j}\left(p_{i}, s_{-j}\right)=Q-\sum_{k \neq j: p_{k}>p_{i}} q_{k}=Q_{i}\left(p_{i}, s_{-i}\right)+q_{j} \geq d_{j}\left(p_{j}\right)+a_{i} .
$$

Consequently, (7) yields $P_{j}\left(d_{j}\left(p_{j}\right)+a_{i}, s_{-j}\right) \leq p_{i}$.

Now express the $\varepsilon$-Nash equilibrium condition for player $j$, with $\tilde{t}=\left(\tilde{q}_{j}, p_{j}\right)$ where $\tilde{q}_{j}:=\min \left(d_{j}\left(p_{j}\right)+a_{i}, d_{j}\left(p_{i}\right)\right)$. The fact that $Q_{j}\left(y, s_{-j}\right)=\lim _{\eta \rightarrow y} \underline{Q}_{j}\left(\eta, s_{-j}\right)$ and the non-decreasingness of $\underline{Q}_{j}\left(., s_{-j}\right)$ imply that $\underline{Q}_{j}\left(p_{j}, s_{-j}\right) \geq d_{j}\left(p_{j}\right)+a_{i}$, so $\tilde{a}_{j}=\tilde{q}_{j}$. Thus we have

$$
\varepsilon \geq \tilde{U}_{j}(\tilde{t})-U_{j}=\theta_{j}\left(\tilde{q}_{j}\right)-\theta_{j}\left(d_{j}\left(p_{j}\right)\right)-\int_{d_{j}\left(p_{j}\right)}^{\tilde{q}_{j}} P_{j}\left(z, s_{-j}\right) \mathrm{d} z .
$$

Using (12) and the non-decreasingness of $P_{j}\left(., s_{-j}\right)$, we obtain

$$
\begin{aligned}
\varepsilon & \geq\left(\theta_{j}^{\prime}\left(\tilde{q}_{j}\right)-p_{i}\right)\left(\tilde{q}_{j}-d_{j}\left(p_{j}\right)\right)+\gamma_{j}\left(\tilde{q}_{j}-d_{j}\left(p_{j}\right)\right)^{2} / 2 \\
& \geq \gamma_{j}\left(\tilde{q}_{j}-d_{j}\left(p_{j}\right)\right)^{2} / 2
\end{aligned}
$$

since $d_{j}\left(p_{j}\right) \leq \tilde{q}_{j} \leq d_{j}\left(p_{i}\right), \theta_{j}^{\prime}\left(d_{j}\left(p_{i}\right)\right)=p_{i}$ and $\theta_{j}^{\prime}$ is non-increasing.

- If $d_{j}\left(p_{i}\right) \geq d_{j}\left(p_{j}\right)+a_{i}$, then $\tilde{q}_{j}=d_{j}\left(p_{j}\right)+a_{i}$, and (22) implies $\varepsilon \geq \gamma_{j} a_{i}^{2} / 2$, But with the $\varepsilon$ chosen, $a_{i} \geq \frac{1}{2} d_{i}(u)$, so we would have $\varepsilon \geq \frac{\gamma_{j}}{8}\left[d_{i}(u)\right]^{2}$, which is in contradiction with the hypotheses.

- Therefore $d_{j}\left(p_{i}\right)<d_{j}\left(p_{j}\right)+a_{i}$, and $\tilde{q}_{j}=d_{j}\left(p_{i}\right)$. (22) then gives $d_{j}\left(p_{i}\right)-$ $d_{j}\left(p_{j}\right) \leq \sqrt{\frac{2 \varepsilon}{\gamma_{j}}}$. Since $0<p_{i}<p_{j}<\theta_{j}^{\prime}(0),(13)$ proves the lemma. 
We can now establish Proposition 3.1.

Proof. If $\left|\mathcal{I}^{+}\right|=1$, then the set $\mathcal{I}^{+}$is reduced to one player $\{i\}$ such that $\theta_{i}^{\prime}(Q)=u$ and $\forall j \in \mathcal{I} \backslash\{i\}, \theta_{j}^{\prime}(0) \leq u$. Lemma 4.2 then gives an upper bound for $p_{i}$. Since no player can ask more quantity than $Q$ and bids are truthful, then $p_{i} \geq u$. We then get $0 \leq p_{i}-u \leq \kappa \sqrt{\frac{2 \varepsilon}{\gamma_{i}}}=C \sqrt{\varepsilon}$, which proves the proposition when $\left|\mathcal{I}^{+}\right|=1$.

Now consider the case $\left|\mathcal{I}^{+}\right|>1$. Lemmas 4.2 and 4.3 prove that for a sufficiently small $\varepsilon$,

$$
|\bar{p}-u| \leq \max \left(1, \frac{1}{\left|\mathcal{I}^{+}\right|-1}\right) \sqrt{2} \kappa \max _{i \in \mathcal{I}^{+}}\left\{\frac{1}{\sqrt{\gamma_{i}}}\right\} \sqrt{\varepsilon}=C \sqrt{\varepsilon}
$$

Moreover, Lemma 4.4 ensures that if there is a player $i \in \mathcal{I}^{+}$such that $p_{i}<u$, then if $\varepsilon$ is sufficiently small,

$$
\max _{i \in \mathcal{I}^{+}}\left(\bar{p}-p_{i}\right) \leq C \sqrt{\varepsilon}
$$

Relations (23) and (24) then yield the proposition.

\section{REFERENCES}

[1] T. Alpcan, T. Başar, R. Srikant and E. Altman, CDMA uplink power control as a noncooperative game. Wireless Networks 8 (2002) 659-670.

[2] S. Baskar, S. Verma, G.S. Tomar and R. Chandra, Auction based bandwidth allocation on the Internet, in Proc. of 3rd IEEE and IFIP International Conference on wireless and Optical Communications Networks (WOCN 2006), Bangalore, India (2006).

[3] F. Beltrán, A note on some properties of an efficient network resource allocation mechanism. Revista de Ingenieria, Facultad de Ingenieria, Universidad de Los Andes (2004).

[4] E.H. Clarke, Multipart pricing of public goods. Public Choice 11 (1971) 17-33.

[5] C. Courcoubetis and R. Weber, Pricing Communication Networks: Economics, Technology and Modelling. Wiley \& Sons, Inc. (2003).

[6] L.A. DaSilva, Pricing for QoS-enabled networks: A survey. IEEE Communications Surveys 3 (2000) 2-8.

[7] T. Groves, Incentives in teams. Econometrica 41 (1973) 617-631.

[8] F.P. Kelly, A.K. Maulloo and D.K.H. Tan, Rate control in communication networks: Shadow prices, proportional fairness and stability. J. Oper. Res. Soc. 49 (1998) 237-252.

[9] A.A. Lazar and N. Semret, Design and analysis of the progressive second price auction for network bandwidth sharing. Telecommunication Systems - Special issue on Network Economics (1999).

[10] J.K. MacKie-Mason and H.R. Varian, Pricing the internet, in Public Access to the Internet, edited by B. Kahin and J. Keller, MIT Press (1995) 269-314.

[11] P. Maillé and B. Tuffin, Pricing the internet with multibid auctions. IEEE/ACM Transactions on Networking 14 (2006) 992-1004.

[12] P. Marbach, Priority service and max-min fairness, in Proc. of IEEE INFOCOM (2002).

[13] R.P. McAfee and J. McMillan, Auctions and bidding. J. Econ. Lit. 25 (1987) 699-738.

[14] R.B. Myerson, Optimal auction design. Mat. Oper. Res. 6 (1981) 58-73. 
[15] A. Pompermaier, A pricing mechanism for intertemporal bandwidth sharing with random utilities and resources. Technical Report LSE-CDAM-2002-06, London School of Economics (2002).

[16] C.U. Saraydar, N.B. Mandayam and D.J. Goodman, Efficient power control via pricing in wireless data networks. IEEE Trans. Comm. 50 (2002) 291-303.

[17] N. Semret, Market Mechanisms for Network Resource Sharing. Ph.D. Thesis, Columbia University (1999)

[18] B. Tuffin, Charging the internet without bandwidth reservation: an overview and bibliography of mathematical approaches. J. Inform. Sci. Engrg. 19 (2003) 765-786.

[19] W. Vickrey, Counterspeculation, auctions, and competitive sealed tenders. J. Finance 16 (1961) 8-37. 